\title{
Pulmonary Valve Disease, CTCAE
}

National Cancer Institute

\section{Source}

National Cancer Institute. Pulmonary Valve Disease, CTCAE. NCI Thesaurus. Code C143793.

A disorder characterized by a defect in pulmonary valve function or structure. 